\section{KEHLER FORUM}

Am 21. Juni 2007, findet von 9:30 bis 17:00 Uhr in der Hochschule Kehl das 14. Kehler Forum statt.

Im Mittelpunkt stehen wieder Strategien und Maßnahmen zur Verwaltungsmodernisierung. Trägerin dieser Veranstaltungsreihe ist die Kehler Hochschule in Zusammenarbeit mit der KommunalBeratung Kehl.

Beim 14. KEHLER FORUM werden folgende fünf Themen in parallel laufenden Arbeitsgruppen behandelt:

1. Zielvereinbarungen als Grundlage für Leistungsentgelte (TVöD-V): Diskussion u.a. folgender Fragen:

- Wie sieht die Zielstruktur einer Kommune aus?

- In welchem Umfang kann eine Kommune in dem vorgegebenen Rahmen eigene Ziele bestimmen?

2. Mitarbeiterbefragungen

- Führungskräftefeedback im Zuge der Einführung einer leistungsorientierten Bezahlung (TVöD)

- Mitarbeiterzufriedenheit und Parameter für das Führungsklima für das Qualitätsmanagement und Balanced Scorecards

3. Einführung des neuen Haushaltsrechts

Eröffnungsbilanz:

- Strategische Überlegungen zur Vermögensbewertung

- Bewertungsvereinfachungen als Kompromiss zwischen Genauigkeit und Machbarkeit

Nachhaltigkeit der Haushaltswirtschaft:

- Nachhaltigkeit als wichtigstes Ziel des neuen Haushaltsrechts

\section{Internet-Portal „NKFW-Netzwerk Bayern“ - Kommunales Wissensportal für das Neue Kommunale Finanzwesen (Doppischer Rechnungsstil)}

Ziel dieser Plattform ist es, für die Kommunen in Bayern und darüber hinaus Synergien bei der Vorbereitung, Umsetzung und Anwendung des Neuen Kommunalen Finanzwesens (Doppik) $\mathrm{zu}$ erzielen bzw. Informationen und Fragestellungen rund um die Doppik bereitzustellen.

Mit dem Beschluss der Innenminister-Konferenz vom November 2003 - dem auch Bayern zugestimmt hat - wurde der Weg für die Einführung eines kaufmännisch orientierten Buchungssystems für Kommunen frei gemacht. $\mathrm{Zu}$ diesem Zeitpunkt befanden sich bereits zwei bayerische „Vorreiter“ im Echtbetrieb einer doppisch geführten Buchhaltung und ein weiterer am Beginn der Umstellungsphase. Zwischenzeitlich - zu Beginn des Jahres 2007 - haben in Bayern ca. 30 Kommunen die Kameralistik durch die kommunale Doppik abgelöst. In den nächsten zwei Jahren wird sich diese Zahl mindestens verdoppeln.

Um allen Kommunen, die an einem Umstieg von der Kameralistik in die kommunale Doppik interessiert sind, diese Erfahrungen und weitere Informationen mit auf den Weg geben zu können, wurde von der Arbeitsgruppe „Doppik“ beim Bayerischen Gemeindetag der Aufbau eines
- Alternativen zur Organisation der Teilhaushalte

- Schlüsselprodukte

- Schlüsselkennzahlen

4. Wege zu einem nachhaltig erfolgreichen Vorschlagswesen

Belebung des Vorschlagswesens und dessen Neubewertung:

- Vorstellung von Good-Practice-Beispielen

- Einfluss von Anreizen und der „Verwaltungskultur“ auf das Vorschlagswesen

- Rahmen für ein Vorschlagswesen

- Abgrenzung des Mitarbeiter-Vorschlagswesens von anderen Ansätzen ab bzw. Ergänzung für andere Ansätze

5. Zentrale IT-Dienstleistungen formulieren - vereinbaren - garantieren

Sicherung der Qualität von IT-Dienstleistungen

- IT Service Management Best-Practice Empfehlungen auf Basis der ITIL (Information Technology Infrastructure Library).

- Die Erfahrungen aus vergleichbaren Situationen in der ÖV, ergänzt durch Referenzen aus Industrie und Handel.

Die Teilnahme kostet 95 Euro. Für Mitglieder des Vereins der Freunde der Hochschule und für Vertreter von Körperschaften, die Mitglied des Vereins sind, kostet die Teilnahme 50 Euro.

Weitere Informationen und Online-Anmeldung:

- Homepage der KommunalBeratung Kehl: www.kommunalberatung-kehl.de

- Homepage der Kehler Hochschule: www.fh-kehl.de.
Informationsportals angeregt. In enger Zusammenarbeit des Bayerischen Staatsministeriums des Innern (BayStMI), der Kommunalen Spitzenverbände und des bayerischen Kommunalen Prüfungsverbandes unter der technischen Federführung der Anstalt für Kommunale Datenverarbeitung in Bayern (AKDB) wurde das gemeinsame Projekt „NKFWNetzwerk Bayern“ realisiert.

Im „NKFW-Netzwerk Bayern“ werden vom BayStMI Informationen aus der Arbeitsgruppe zur Reform des Kommunalen Haushaltsrechts, die mit den Kommunalen Spitzenverbänden und dem Bayerischen Kommunalen Prüfungsverband abgestimmt sind, zur Verfügung gestellt. Darüber hinaus werden auch Beiträge jedes einzelnen Verbandes angeboten.

Kommunen, die bereits umgestellt haben bzw. auf dem Weg in die kommunale Doppik sind, wird die Möglichkeit geboten, ihre Umstellungsprozesse und Erfahrungen $\mathrm{zu}$ präsentieren. Informationen der Vorreiter und weiterer Kommunen können bereits über das NKFW-Netzwerk Bayern abgerufen werden.

Hinweise zu Aus- und Fortbildungsmöglichkeiten und Veranstaltungen öffentlicher Anbieter zum Thema „Reformen im kommunalen Finanzwesen" runden das Informationsangebot ab. Viele der angebotenen Informationen werden auch als Download-Version zur Verfügung gestellt. 


\section{Neue Organisationsformen für Regierung und Verwaltung auf IT-Basis}

Hochschule Wismar und Institute for eGovernment schlossen Kooperationsvereinbarung

Die Hochschule Wismar und das Institute for eGovernment (IfG.CC), eine eingetragene Vereinigung an der Universität Potsdam, haben eine auf fünf Jahre ausgelegte Kooperationsvereinbarung abgeschlossen. Ziel der Wissenschaftler aus Wismar und Potsdam ist es, auf den Erfahrungen aus gemeinsamen Projekten aufbauend, die Zusammenarbeit zu vertiefen. Dazu gehört der Austausch von wissenschaftlichem Personal und Studierenden. Gegenstand der Kooperation ist die Zusammenarbeit im Bereich der Lehre und die Durchführung von Forschungsprojekten, die insbesondere den Ostseeraum betreffen. Die Hochschule Wismar, die im nächsten Jahr ihr 100-jähriges Bestehen feiern wird, bringt in die Kooperation insbesondere Wissen und Forschungserfahrung auf dem Gebiet der Wirtschaftsinformatik ein. Die Mitarbeiter des Potsdamer Institute for eGovernment verfügen über Erfahrungen in der Forschung auf den Gebieten von eGovernment und typischer Informatik im Verwaltungsbereich.

„Beide Institutionen besitzen langjährige Erfahrungen in der Bearbeitung von internationalen Forschungsprojekten und arbeiten zurzeit gemeinsam im INTERREG-Projekt „ASAP“ zusammen. Dieses widmet sich der Verbesserung der Verwaltungsstrukturen im ländlichen Raum. Es ist geplant, sich zukünftig gemeinsam an EU-Projekten im Ostseeraum zu beteiligen“, so Dr. Tino Schuppan, Direktor des IfG.CC. Dabei sollen neue Organisationsformen auf IT-Basis erprobt werden. „Wir sind dabei, die Rolle der Hochschule Wismar im Bereich der Regionalentwicklung zu verstärken. Daher hat für uns die strategische Zusammenarbeit mit dem IfG.CC in Potsdam einen hohen Stellenwert. Denn durch die Kooperation bietet sich die Möglichkeit, die Kompetenzen des IfG.CC im Bereich
eGovernment zu nutzen“, so Prof. Dr. Gunnar Prause, Prodekan an der Fakultät für Wirtschaftswissenschaften der Hochschule Wismar. Gerade für Mecklenburg-Vorpommern und die neuen EU-Mitgliedsländer, für die das Thema der IT-Nutzung im ländlichen Raum eine besondere Bedeutung besitzt, eröffnen die eGovernment-Erfahrungen des IfG.CC eine Vielzahl von neuen Projekt- und Forschungsmöglichkeiten.

\section{E-Government in medias res - E-Government- Konferenz}

Die Konferenz findet am 12./13. Juli 2007 in Bremen (Haus der Wissenschaft und der Bremischen Bürgerschaft) statt. Während der Konferenz werden die praktischen Probleme und der gegenwärtige Sachstand der Umsetzung von EGovernment vorgestellt und diskutiert.

Eröffnet wird die Konferenz u.a. mit Vorträgen von Matthias Kammer, dataport, Prof. Dr. Herbert Kubicek und Dr. Martin Wind, beide Institut für Informationsmanagement Bremen (ifib).

Es gibt sechs Fachforen zu folgenden Themen:

- Zentralregister und Identity Management,

- Deutschland Infrastruktur,

- GIS in der Virtuellen Region Nordwest,

- EU-Dienstleistungsrichtlinie,

- Standardisierung

- Informationsmanagement/Informationsfreiheit/Barrierefreiheit.

Die Teilnahme an der Konferenz ist kostenlos.

Weitere Informationen und Anmeldung:

www.finanzen.bremen.de/info/inmediasres.

\section{Redaktionsanschrift:}

Prof. Dr. Veith Mehde (V.i.S.d.P.), Juristische Fakultät der Leibniz

Universität Hannover

Königsworther Platz 1 | 30167 Hannover

Tel. (0511) 762 - 8206 | Fax (0511) 762 - 19106

E-Mail: mehde@jura.uni-hannover.de | www: http://www.verwaltung-

management.de

Redaktionsassistentin (Satz und Layout):

Heidrun Müller, Institute for eGovernment (IfG.CC) an der Universität Potsdam

\section{Druck und Verlag:}

Nomos Verlagsgesellschaft mbH \& Co. KG

Waldseestraße 3-5 | D-76530 Baden-Baden

Telefon (07221) 2104-O | Fax (07221) 2104-27

\section{Anzeigen:}

sales friendly Verlagsdienstleistungen | Siegburger Str. 123 | 53229 Bonn

Telefon (0228) 978980 | Fax (0228) 9789820

E-Mail:roos@sales-friendly.de
Die Zeitschrift, sowie alle in ihr enthaltenen einzelnen Beiträge und Abbildungen sind urheberrechtlich geschützt. Jede Verwertung, die nicht ausdrücklich vom Urheberrechtsgesetz zugelassen ist, bedarf der vorherigen Zustimmung des Verlags.

Namentlich gekennzeichnete Artikel müssen nicht die Meinung der Herausgeber/Redaktion wiedergeben. Unverlangt eingesandte Manuskripte - für die keine Haftung übernommen wird - gelten als Veröffentlichungsvorschlag zu den Bedingungen des Verlages. Es werden nur unveröffentlichte Originalarbeiten angenommen. Die Verfasser erklären sich mit einer nicht sinnentstellenden redaktionellen Bearbeitung einverstanden.

Erscheinungsweise: sechsmal jährlich

Bezugspreis 2007: jährlich 120,- Euro, Einzelheft 24,- Euro. Alle Preise zzgl. Vertriebs-/Direktbeorderungsgebühren inkl. Mwst.; Bestellungen nehmen entgegen: Der Buchhandel und der Verlag; Abbestellungen mit Drei-Monats-Frist zum Kalenderjahresende. Zahlungen jeweils im Voraus an: Nomos Verlagsgesellschaft, Postbank Karlsruhe, Konto 73636751 (BLZ 660 100 75) und Stadtsparkasse Baden-Baden, Konto 5-002266 (BLZ 66250030$)$.

ISSN $0947-9856$ 